secondary mechanisms to enhance infant survival. The characteristic presence of parental behaviour in birds and mammals (associated with vivipary in the latter) may thus be linked to the presence of endothermy.

Going beyond the material presented by Bennett and Ruben, it is also noteworthy that there is probably a close connection between metabolic turnover, activity levels, aerobic capacity and brain size in birds and mammals. The fact that birds and mammals have relatively larger brains than reptiles, amphibians and fish (Jerison Evolution of the Brain and Intelligence, 1973) could relate directly to greater locomotor capacity and to the high metabolic levels associated with endothermy. If a single such explanation covering all these aspects is applicable to living birds and mammals, these corollaries (and others as yet unidentified) could considerably increase our understanding of vertebrate evolution. The present controversy over the presence or absence of endothermy in dinosaurs, having stimulated the search for new explanatory principles, may itself find an acceptable solution within this unitary framework, bringing in such topics as relative brain size (Hopson op.cit., 1977; Jerison op.cit., 1973) and evidence for parental behaviour in dinosaurs (Hopson op. cit. 1973; Horner \& Makela Nature 282, 296; 1979).

\section{Switchboards and folds}

\section{from P.D. Calvert}

SEMICRYSTALline polymers derive their useful properties of strength and toughness from their structure of alternating crystalline and rubbery layers. Hence the way in which the chains are arranged in these layers and bond them together is of importance in guiding our thinking about many aspects of their behaviour.

Small angle neutron scattering (SANS) results on crystalline polyethylene published in 1976 showed that current ideas on the arrangement of the molecular chains in crystalline polymers needed rethinking (see News and Views 263, 371; 1976). Polymer crystals are usually thin, about $10 \mathrm{~nm}$, in the direction traversed by the chains so it is agreed that chains must fold back for several traverses through any crystal. Before 1976 it was widely believed that the chains were folded tightly to give adjacent re-entry with little loose material at the crystal surface. The alternative was the 'telephone switchboard' model where the chains re-entered the crystal at some distance from their exit point. The Faraday Discussion on 'Organisation of Macromolecules in the Condensed Phase' held in Cambridge last autumn gave a clear picture of developments over the past 3 years.

The basic observation by neutron scattering, that there is little change in the radius of gyration of polyethylene on crystallisation, was originally reported by Ballard and coworkers at ICI. They have since shown that polypropylene behaves similarly and workers at Bristol and at Mainz have confirmed the polyethylene result. At Cambridge, J. Guenet, C. Picot and $\mathrm{H}$. Benoit from Strasbouig reported similar results for deuterated polystyrene chains in a high molecular weight $\left(M_{w}=1.7 \times 10^{6}\right)$ polystyrene matrix. However, in a matrix of lower molecular weight $\left(\mathrm{M}_{w}=4 \times 10^{5}\right)$ the radius of gyration increased by up to $40 \%$ on crystallisation. This is of great interest because the fact that no change in radius of gyration is usually observed means that there is little with which really to test theories. One problem with SANS is that segregation of the labelled molecules may occur on crystallisation, but the Strasbourg group claims that this is not so in their samples.

Several different approaches have been successful in fitting the SANS data. D. Yoon and P. Flory (Stanford University) have shown that switchboard models with or without some low probability of adjacent re-entry can fit the data. The Mainz group has used a 'solidification' model in which straightening of chain sections is superimposed on the initial, molten random coil. The Strasbourg group has fitted its data by allowing adjacent reentry over the central sections of the chain and leaving amorphous 'wings' at either end. The US National Bureau of Standards group here had success with this and with a cluster model, where clusters of three or four adjacent folds are linked by longer loose folds. The lesson seems to be that the many different models can be fitted to the data available so far, which can only be used to eliminate some possibilities. However, matters should improve as measurements are extended up to 'intermediate' angles where the scattering vector is commensurate with the individual folds and clusters rather than the whole chain. Careful measurements of absolute, rather than relative, intensity may also be necessary in these regions.

The ICI/Julich group has looked carefully at the behaviour of the radius of gyration at low molecular weights in polypropylene (Polymer 20, 399; 1979). In principle, the chain might be expected to resemble a straight rod when it becomes so short that it can only fold once or twice. Then the rod length should be equal to the lamellar spacing seen by small angle X-ray scattering. In fact it is about twice this. This they have explained in terms of chains running through pairs of lamellae. However, we are rather ignorant about the way in which the stacks of lamellar crystals form; it often seems to be a two-step process with later filling-in by small lamellae of the spaces between those which form first. Thus it is difficult to see how really to view this interesting result. This group also reported data on drawn polypropylene where a six-fold draw ratio resulted in a decrease of 3-4 times in the radius of gyration perpendicular to the draw direction, more than the decrease of $\sqrt{6}$ that might be expected. There was also a decrease of 3-6 times in the molecular weight measured by SANS although the chains were unbroken suggesting that the chain was now acting as a series of separate beads.

Given that interpretation of the SANS data on melt-grown crystals is very dependent on the models used, one would hope for signs of theoretical advances. Here the meeting at Cambridge had little to offer. The interpretation of the results of other experimental observations in terms of the switchboard and adjacent re-entry models were debated, as they have been for many years, but little progress has been made. A great deal of information exists but most of it can be interpreted in terms of more than one model. However, it still remains unclear whether the telephone switchboard model can be reconciled with sensible packing densities close to the crystal surface. Recently Flory suggested that there was not time for a full adjacent re-entry conformation to be adopted during crystallisation, given the relaxation time of a whole chain. J. Klein and E. Di Marzio argued against this, the former by calculating the diffusion rate of a chain in a tube and the latter by calculating the time to pull a chain through a hole in a plane, but as all the calculations are on different bases it is difficult to grasp the reasons for the contradictions. A telling point is that polymeric impurities can be pushed aside even during rapid crystallisation. It is worth giving more thought to the relationship between the time required for this process and that for crystallisation.

Studies on solution-grown polyethylene single crystals seemed to have reached a reasonable level of agreement. Yoon, D. Sadler (Bristol) and M. Stamm (Mainz) all talked in terms of chains re-entering close to their point of exit and spread over a few neighbouring crystal planes, in other words not 'adjacent' re-entry but 'nearby' reentry. However, there are still differences in the form of the models used by the different groups.

The SANS results have changed most people's picture of the crystal surface. They now picture it with many loose loops whereas previously the majority viewpoint was that the surface was virtually smooth. For the future we must await more detail from the neutron scattering at intermediate angles. The most viable theoretical approach seems to me to be to start from the molten random coil and to interpret the action of the kinetics and thermodynamics of crystallisation and annealing but progress has been slow so far.

Paul Calvert is a lecturer in the School of Molecular Sciences, University of Sussex. 